\title{
Identification of a Gene Encoding Glutamate Decarboxylase Involved in the Postharvest Fruit Ripening Process in Banana
}

\author{
Wei Hu, Ju-Hua Liu, Xiao-Ying Yang, Jian-Bin Zhang, Cai-Hong Jia, \\ Mei-Ying Li, and $\mathrm{Bi}-\mathrm{Yu} \mathrm{Xu}{ }^{1}$ \\ Key Laboratory of Biology and Genetic Resources of Tropical Crops, \\ Institute of Tropical Bioscience and Biotechnology, Chinese Academy of \\ Tropical Agricultural Sciences, Xueyuan Road 4, Longhua County, Haikou \\ City, Hainan Province 571101, People's Republic of China
}

\section{Zhi-Qiang Jin ${ }^{1}$}

Key Laboratory of Biology and Genetic Resources of Tropical Crops, Institute of Tropical Bioscience and Biotechnology, Chinese Academy of Tropical Agricultural Sciences, Xueyuan Road 4, Longhua County, Haikou City, Hainan Province 571101, People's Republic of China; and Hainan Key Laboratory of Banana Genetic Improvement, Haikou Experimental Station, Institute of Banana, Chinese Academy of Tropical Agricultural Sciences, Yilong W Road 2, Longhua County, Haikou City, Hainan Province 570102, People's Republic of China

Additional index words. banana (Musa acuminata L. AAA group cv. Brazilian), ethylene biosynthesis, fruit ripening, glutamate decarboxylase, postharvest

\begin{abstract}
The banana, a typical climacteric fruit, undergoes a postharvest ripening process followed by a burst in ethylene production that signals the beginning of the climacteric period. Postharvest ripening plays an important role in improving the quality of the fruit as well as limiting its shelf life. To investigate the role of glutamate decarboxylase (GAD) in climacteric ethylene biosynthesis and fruit ripening in postharvest banana, a GAD gene was isolated from banana, designated $M u G A D$. Coincidently with climacteric ethylene production, $M u G A D$ expression as well as the expression of the genes encoding the Musa 1-aminocyclopropane-1-carboxylate synthase (MaACS1) and Musa 1-aminocyclopropane-1-carboxylate oxidase (MaACO1) greatly increased during natural ripening and in ethylene-treated banana. Moreover, ethylene biosynthesis, ripening progress, and $M u G A D, M a A C S 1$, and $M a A C O 1$ expression were enhanced by exogenous ethylene application and inhibited by 1-methylcyclopropene (1MCP). Taken together, our results suggested that $M u G A D$ is involved in the fruit ripening process in postharvest banana.
\end{abstract}

The enzyme GAD (EC 4.1.1.15) is responsible for converting L-glutamic acid to gamma-aminobutyric acid (GABA) in the presence of the cofactor pyridoxal 5'-phosphate (PLP). GABA, a non-protein amino acid, is ubiquitously present in prokaryotes and eukaryotes. Plant GADs carry a $\mathrm{C}$-terminal extension that binds to $\mathrm{Ca}^{2+} /$ calmodulin (CaM) to modulate enzyme activity (Chen et al., 1994; Ling et al., 1994).

Received for publication 10 Dec. 2013. Accepted for publication 13 Apr. 2014.

This work was supported by the earmarked fund for Modern Agro-industry Technology Research System (CARS-32), the Ministry of Science and Technology of the People's Republic of China (2011AA10020650), and the Major Technology Project of Hainan (ZDZX2013023-1).

${ }^{1}$ To whom reprint requests should be addressed; e-mail biyuxu@126.com; 18689846976@163.com.
Changes in gene expression and/or activity of GADs in plants are regulated by cytosolic levels of $\mathrm{H}^{+}$and $\mathrm{Ca}^{2+}$ that are associated with various environmental stimuli (Bown and Shelp, 1989), including mechanical stimuli (Wallace et al., 1984) or damage (Ramputh and Bown, 1996), cold shock (Mazzucotelli et al., 2006), heat shock (Mayer et al., 1990), hypoxia (Tsushida and Murai, 1987), cytosolic acidification (Crawford et al., 1994), water stress (Bolarin et al., 1995), and phytohormones (Ford et al., 1996). Activated GADs lead to GABA accumulation that is important in regulating cytoplasmic acidity, glucose use, nitrogen storage, plant development, plant defense, and fruit ripening (Bown and Shelp, 1997; Gallego et al., 1995).

Although GAD and its product GABA have been identified in legumes decades ago, their functional role in plants, especially in ethylene biosynthesis and fruit ripening, is poorly understood (Kulkarni and Sohonie,
1956). Previous studies investigating the role of GADs in fruit ripening isolated GAD genes in several species of fruits, including tomato and citrus. These studies showed that the expression of GAD genes is changed at different ripening stages (Akihiro et al., 2008; Cercós et al., 2006; Gallego et al., 1995). Furthermore, the relationship between GABA levels and ethylene production was investigated in excised sunflower tissues to elucidate the role of GAD in ethylene biosynthesis. The results indicated that exogenous GABA caused an increase in ethylene production rate mainly by promoting GAD activity and up-regulating 1-aminocyclopropane-1-carboxylate (ACC) synthase (ACS: EC 4.4.1.14) gene expression (Kathiresan et al., 1997). However, the function of GAD in climacteric ethylene biosynthesis and postharvest fruit ripening is unclear.

The banana (Musa acuminata L. AAA group cv. Brazilian), a typical climacteric fruit, undergoes a postharvest ripening process characterized by a green storage phase followed by a burst in ethylene production that signals the beginning of the climacteric period. The banana enzymes involved in ethylene biosynthesis have been well characterized, and the related cDNAs were cloned. For example, MaACS1, encoding ACS, and MaACO1, encoding ACC oxidase (ACO: EC 1.4.3), were shown to play crucial roles in ethylene biosynthesis in banana postharvest ripening (Adams and Yang, 1979; Liu et al., 1999; Yang and Hoffman, 1984). Coincidently with the respiratory climacteric period, numerous physiological and biochemical changes occur in the banana fruit, including chlorophyll breakdown, flavochrome accumulation (Jacob-Wilk et al., 1999; Thomas and Janave, 1992), degradation of cell wall components resulting in fruit softening (Lohani et al., 2004), and degradation of stored starch into soluble sugar (Hill and Rees, 1995a, 1995b). These changes influence characteristics of the banana fruit such as its firmness, astringency, aroma, color, and shelf life. As a result, postharvest ripening plays an important role in improving the quality of the fruit as well as limiting its shelf life.

In our previous study (Xu et al., 2007), we constructed a forward suppression subtractive hybridization (SSH) cDNA library. Specifically, $265 \mathrm{cDNAs}$ were isolated that were up-regulated in the ripening banana at $2 \mathrm{~d}$ postharvest (DPH). To investigate the effects of these differentially expressed cDNAs in the initiation and peaking of climacteric ethylene biosynthesis after harvest, cDNA microarray analysis was used to identify up- or down-regulated cDNAs at the onset of climacteric ethylene biosynthesis. A cDNA encoding glutamate decarboxylase was up-regulated during climacteric ethylene biosynthesis. Moreover, the expression level of this cDNA was the highest among the levels of all the cDNAs in the microarray on the initiation of ethylene biosynthesis (Jin et al., 2009; Xu et al., 2007). Our previous evidences implied that the glutamate decarboxylase activity might be related to ethylene biosynthesis associated with banana postharvest ripening. Therefore, we isolated the full-length cDNA 
of the glutamate decarboxylase gene from banana $(M u G A D)$.

In this study, by studying changes in the ethylene production and expression of $M u G A D$, $M a A C S 1$, and $M a A C O 1$ of banana fruits related to ripening under various treatment conditions, we investigated the role of $M u G A D$ in post-harvest ripening. Our results showed that $M u G A D$ expression correlates with the expression of MaACS1 and MaACO1, key genes in ethylene biosynthesis.

\section{Materials and Methods}

Plant materials and treatments. Hands of mature, unripe bananas (Musa acuminata L. AAA group cv. Brazilian) were obtained from the banana plantation of the Institute of Tropical Bioscience and Biotechnology (Chengmai, Hainan). Fruits from the same hand were harvested and washed with distilled water. Banana hands were separated into individual fingers representing the same developmental stage (Inaba and Nakamura, 1986). The degree of ripening was classified into seven stages according to Pua et al. (2003), namely full green (FG), trace yellow (TY), more green than yellow (MG), more yellow than green (MY), green tip (GT), full yellow (FY), and yellow flecked with brown spots (YB). The YB stage is the most advanced stage, when bananas have completely ripened during the postharvest ripening process.

Banana fingers were divided into three sample groups, including naturally ripened, ethylene-treated, and 1-MCP-treated. Each sample represented three replicates (each replicate had three banana fingers). The samples were collected to perform physiological experiments according to ripening stages. For natural ripening, bananas were allowed to ripen naturally at $25{ }^{\circ} \mathrm{C}$. For ethylene treatment, bananas were treated with $100 \mu \mathrm{L} \cdot \mathrm{L}^{-1}$ ethylene for $18 \mathrm{~h}$ and then ripened naturally at $25{ }^{\circ} \mathrm{C}$. For the 1-MCP treatment, bananas were kept in an airtight container with $1 \mu \mathrm{L} \cdot \mathrm{L}^{-1} 1-\mathrm{MCP}$ for $24 \mathrm{~h}$ and then ripened at $25^{\circ} \mathrm{C}$, as described previously (Lohani et al., 2004). At every ripening stage, ethylene production of each treatment group was measured, and banana pulp was frozen in liquid nitrogen and stored at $-80^{\circ} \mathrm{C}$ for total RNA extraction. Total RNA was isolated from fruit pulp tissues of naturally ripened, ethylene- and 1-MCP-treated bananas using the method described by Wan and Wilkins (1994).

Measurement of ethylene production. Ethylene production was measured by enclosing fruit samples in an airtight container for $2 \mathrm{~h}$ at $25^{\circ} \mathrm{C}$, withdrawing $1 \mathrm{~mL}$ of the headspace gas, and injecting it into a gas chromatograph (GC-2010; Shimadzu, Kyoto, Japan) fitted with a flame ionization detector and an activated alumina column. The ethylene production measurements were performed as recommended by the manufacturer.

Cloning and sequencing. Rapid amplification of cDNA ends (RACE) was used to obtain the full-length cDNA of $M u G A D$ based on the partial sequence obtained by
SSH previously (Xu et al., 2007). 5' - and $3^{\prime}$-RACE were conducted using the SMART PCR cDNA Synthesis Kit (Clontech, Palo Alto, CA) with the double-stranded cDNA from 8-DPH banana fruit as a template. For 5 '-RACE, the forward and reverse primer pair was P1. For 3'-RACE, the forward and reverse primer pair was $\mathrm{P} 2$ (Table 1).

Fragments of MaACO1, MaACS1, and MaActin1 were cloned using the doublestranded cDNA from 8-DPH banana fruit as a template. The forward and reverse primers were designed according to GenBank as well as the standards for the real-time reverse transcriptase-polymerase chain reaction (real-time RT-PCR; TaKaRa Biotechnology, Dalian, China). To clone the $M a A C O 1$ fragment, the forward and reverse primer pair was $\mathrm{P} 3$ designed according to the GenBank sequence (accession no. AJ223232). To clone the MaACS1 fragment, the forward and reverse primer pair was $\mathrm{P} 4$ (accession no. AB021906). To clone the MaActin1 fragment, the forward and reverse primer pair was P5 (accession no. AF285176). All the fragments were cloned into the PMD-18T (TaKaRa) vector and sequenced. Based on the $5^{\prime}-$ and $3^{\prime}$-end sequences of the $M u G A D \mathrm{cDNA}$, a pair of primers was designed P6 to allow amplification of the entire open reading frame (Table 1). The amplified product was inserted into the PMD-18T vector and sequenced. The sequences were compared with those in the National Center for Biotechnology Information database using BLAST $(<\mathrm{http}: / / \mathrm{www}$. ncbi.nlm.nih.gov/BLAST/>) and DNAMAN software.

Real-time RT-PCR. The first strand of cDNA was synthesized using AMV reverse transcriptase (Promega) from $5 \mu \mathrm{g}$ RNA in a $20-\mu \mathrm{L}$ reaction volume, which subsequently was used as the template in real-time RT-PCR. For the amplification of $M u G A D, M a A C O 1$, MaACS1, and MaActin1 cDNA fragments, the primers that were listed in cloning and RT-PCR sections were designed according to the TaKaRa real-time RT-PCR instructions. The primers did not contain the highly conserved protein domains and had high efficiency and specificity, which was determined using the MxProTM real-time RT-PCR software (Stratagene, CA) and agarose gel electrophoresis. The resulted real-time RT-PCR products for $M u G A D, M a A C O 1, M a A C S 1$, and MaActinl were $175,151,127$, and 379 $\mathrm{bp}$, respectively, which were suitable for real-time RT-PCR.

SYBR Premix Ex TaqTM (TaKaRa, Dalian, China) was used in a $25-\mu \mathrm{L}$ reaction volume with $0.5 \mu \mathrm{L}$ ROX reference dye. Primers were used at $100 \mathrm{nM}$ concentrations with the reverse-transcribed RNA template per reaction. In all experiments, appropriate negative controls lacking template RNA were subjected to the same procedure to detect possible contamination. Before proceeding with the actual experiments, a series of template and primer dilutions were tested to determine the optimal concentrations for maximal target amplification. Amplification efficiencies for the internal control and target genes were between 0.9 and 0.97 .

Each quantitative real-time PCR was performed on a Stratagene Mx3000P (Stratagene) machine using SYBR chemistry. The proper thermal cycling conditions for $M u G A D$, MaACS1, and MaActin 1 were $94^{\circ} \mathrm{C}$ for $3 \mathrm{~min}$ followed by 40 cycles at $94{ }^{\circ} \mathrm{C}$ for $7 \mathrm{~s}, 55^{\circ} \mathrm{C}$ for $10 \mathrm{~s}$, and $72^{\circ} \mathrm{C}$ for $15 \mathrm{~s}$; and for $M a A C O 1$, conditions were $94{ }^{\circ} \mathrm{C}$ for $3 \mathrm{~min}$ followed by 40 cycles at $94{ }^{\circ} \mathrm{C}$ for $7 \mathrm{~s}$, and $60^{\circ} \mathrm{C}$ for $20 \mathrm{~s}$. Reactions were performed in triplicate, and data were analyzed using the MxProTM real-time RT-PCR software (Stratagene). MaActinl was used as a control sample that served as a benchmark to which the $M u G A D$, $M a A C O 1$, and MaACS1 products were normalized. The differences in $\mathrm{Ct}$ values among the MuGAD, MaACO1, MaACS1, and MaActin1 transcripts were expressed as fold-changes relative to MaActin 1. mRNA-fold difference was calculated relative to the control and relative expression level of genes was calculated using the $2^{-\Delta \Delta \mathrm{Ct}}$ formula (Livak and Schmittgen, 2001).

\section{Results}

Cloning and sequence analysis of $\mathrm{MuGAD}$, MaACO1, MaACS1, and MaActin1. In our previous study, a cDNA fragment sharing high homology to known GAD genes from other plant species was obtained using $\mathrm{SSH}$ (Xu et al., 2007). Here, we isolated the full-length cDNA, designated as $M u G A D$, from banana fruit at $8 \mathrm{DPH}$ using RACE. Sequence analysis indicated that the $M u G A D$ cDNA is 1771 bp long with a 1500 -bp open reading frame. The predicted MuGAD protein contained 499 amino acids with a molecular mass of $56.25 \mathrm{kDa}$. BlastX analysis showed that the predicted MuGAD protein shared a high degree of sequence similarity with GAD proteins from other plant species, including $81 \%$ identity with OsGAD from Oryza sativa, $80 \%$ with CsGAD from Citrus sinensis and PtGAD from Populus tremula, and $78 \%$ identity with SIGAD from Solanum lycopersicum. Furthermore, the MaACS1, MaACO1, and MaActin1 fragments shared high sequence identity to AB021906 (99\%), AJ223232 (100\%), and AF285176 (100\%)

Table 1. Primers used for polymerase chain reaction analysis.

\begin{tabular}{lcll}
\hline Gene & Primer & \multicolumn{1}{c}{ Forward primer $\left(5^{\prime}-3^{\prime}\right)$} & \multicolumn{1}{c}{ Reverse primer $\left(5^{\prime}-3^{\prime}\right)$} \\
\hline$M u G A D$ & P1 & $5^{\prime}$-ggaagtcccactctagttcaggatag-3' & $5^{\prime}$-ctccgagatctggacgagc-3' \\
$M u G A D$ & P2 & $5^{\prime}$-atctggatctggtggcgaagtttg-3' & $5^{\prime}$-taatacgactcactcactataggg-3' \\
$M a A C O 1$ & P3 & $5^{\prime}$-agaaggaagcggagga-3' & $5^{\prime}$-gtagcgattgggtggg-3' \\
$M a A C S 1$ & P4 & $5^{\prime}$ - aagaggaggtgggacg-3' & $5^{\prime}$-cgatgttcaggtggc-3' \\
$M a A c t i n 1$ & P5 & $5^{\prime}$-cgaggctcaatcaaaga-3' & $5^{\prime}$-accagcaaggtccaaac-3' \\
$M u G A D$ & P6 & $5^{\prime}$-gatgactctctcggcggtagcat-3' & $5^{\prime}$-gataccaccaacggaaccccaaat-3' \\
\hline
\end{tabular}


in Musa acuminata, respectively, suggesting that the cloned cDNAs were indeed the genes that encode ACS, ACO, and actin, respectively.

Amino acid sequence alignment indicated that MuGAD contained a CaM-binding domain at its C-terminus, a putative PLP-binding motif, and the GAD active domain (Akama et al., 2001) (Fig. 1). In addition, a phylogenetic tree was constructed based on the amino acid sequence alignment of MuGAD and 11 other plant GADs from GenBank. The resulting dendrogram showed that MuGAD was very close to SIGAD (Fig. 2). These results suggested that $M u G A D$ obtained in this study is a novel $G A D$ gene from banana.

Ethylene biosynthesis and differential expression of MuGAD, MaACO1, and MaACS1 at different stages of banana ripening. To investigate the relationship between endogenous ethylene biosynthesis associated with postharvest banana ripening and the expression of $M u G A D$, exogenous ethylene and 1-MCP that have a profound effect on fruit ripening were used to treat banana fruits. Changes in the rate of endogenous ethylene production, timing of ripening stages as well as $M u G A D, M a A C O 1$, and MaACS1 expression were examined at different banana ripening stages (Figs. 3 and 4).

In naturally ripened bananas, endogenous ethylene production was low between $0 \mathrm{DPH}$ (FG stage; 1.7) and 10 DPH (MY stage; 5.0) and then increased greatly at $12 \mathrm{DPH}$ (GT stage; 7.2) and peaked at 15 DPH (FY stage; 20.7) followed by a sharp decrease at $20 \mathrm{DPH}$ (YB stage; 3.4). A sharp 4-fold increase in endogenous ethylene production (from 5.0 to 20.7) occurred between the MY and FY stages (Fig. 3A). It took bananas $20 \mathrm{~d}$ after harvest to reach the most advanced $\mathrm{YB}$ stage (Fig. 3A). Paralleling these ripening events, the expression of $M u G A D$ and MaACS1 was detectable during the preclimacteric period, then increased from $10(\mathrm{MY})$ to $15 \mathrm{DPH}$ (FY), and reached the maximum level at 15 DPH (FY), coincidental with the highest level of endogenous ethylene production (Figs. 4A and 4C). In fact, an 11-fold sharp increase in $M u G A D$ expression (from 3.6 to 40.1) occurred between 10 (MY) and $15 \mathrm{DPH}$ (FY) (Fig. 4A). Similarly, a 12-fold sharp increase in MaACS1 expression (from 371.3 to 4508.2 ) occurred between 10 (MY) and 15 DPH (FY) (Fig. 4C). Although MaACO1 expression was lower compared with the expression levels of MaACS1, the expression of $\mathrm{MaACO} 1$ increased from the onset of the climacteric period (from 2.3 to 20.9) and increased 11-fold between 10 (MY) and 15 DPH (FY) (Fig. 4B).

Endogenous ethylene production, ripening progress, and expression of $M u G A D$, $M a A C O 1$, and MaACS1 were enhanced in bananas treated with exogenous ethylene (Figs. 3 and 4). First of all, endogenous ethylene biosynthesis was activated immediately after ethylene treatment, strongly increased at 2 DPH (MG; 16.9), and peaked at 3 DPH (MY; 29.0). This was a remarkable advance in ethylene production $12 \mathrm{~d}$ before the highest
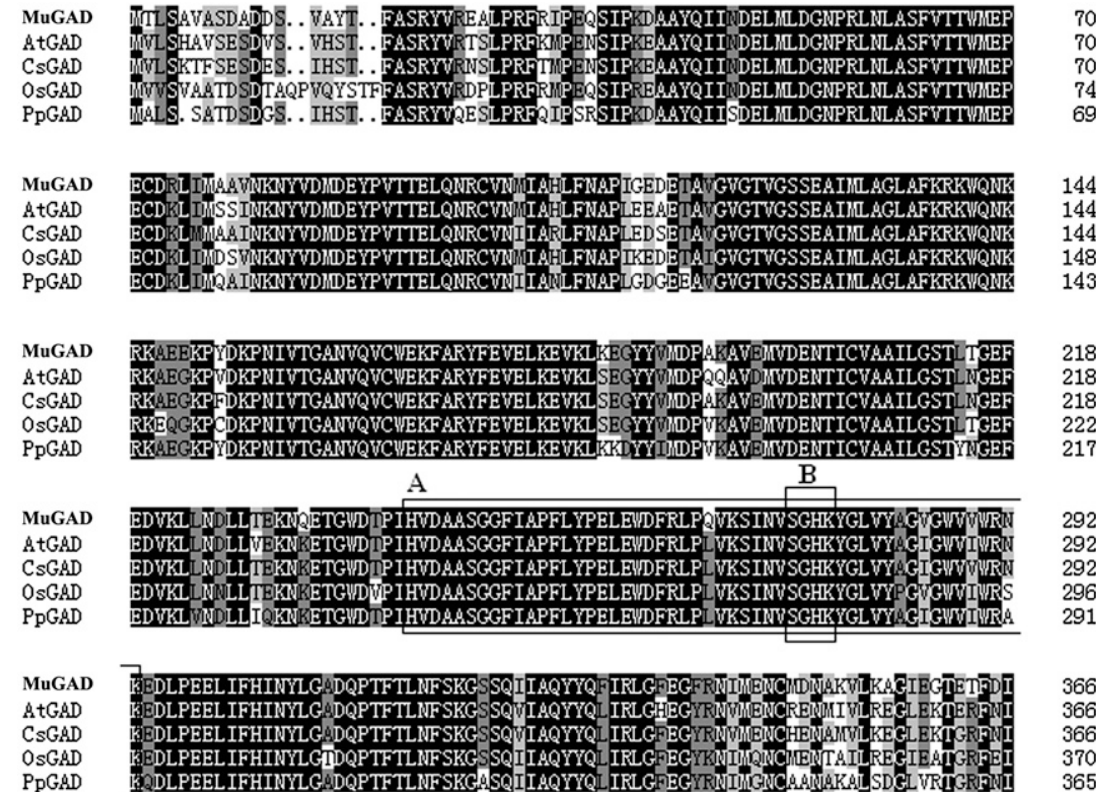

PpGAD

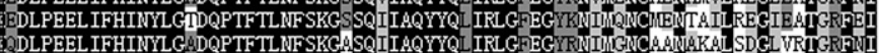

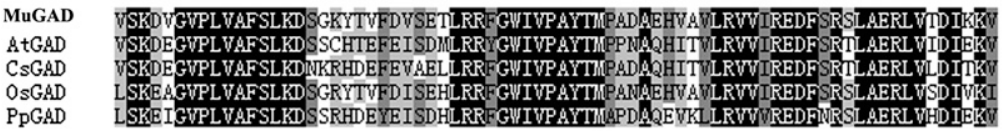

440

440

440 439

MuGAD

AtGAD

CSGAD

OsGAD

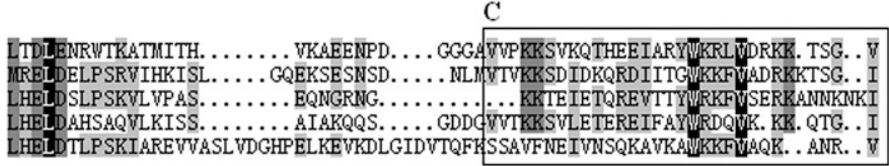

498

501

493

500

Fig. 1. Alignment of the predicted MuGAD sequence with sequences of glutamate decarboxylase (GAD) homologs from other plant species. The sequences from Arabidopsis thaliana AtGAD (NP_197235), Citrus sinensis CsGAD (AAZ05070), Oryza sativa Japonica Group OsGAD (BAB32868), and Pinus pinaster PpGAD (ABA18653) are aligned with MuGAD (this study) using DNAMAN software. (A) GAD active domain. (B) Consensus motif for pyridoxal 5' -phosphate binding. (C) Calmodulin (CaM)-binding domain.

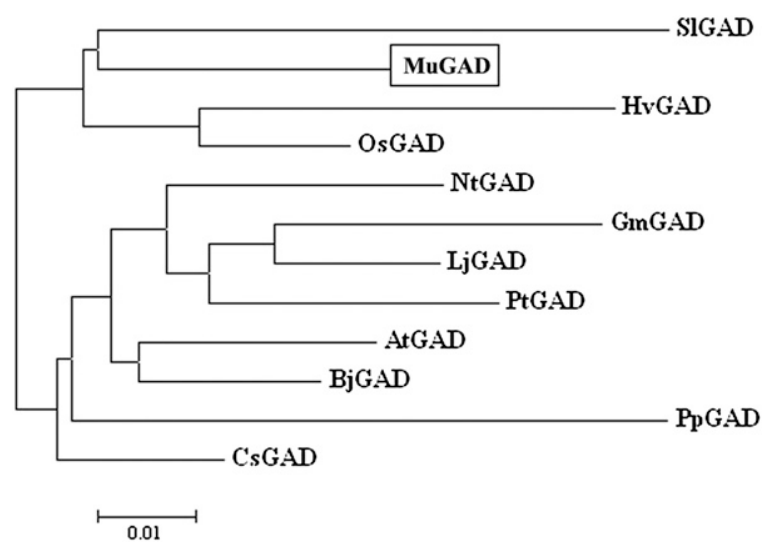

Fig. 2. Phylogenetic tree of glutamate decarboxylases (GADs) from various plants generated by Clustal W. GenBank accession numbers: MuGAD, Musa acuminata (boxed, this study); SIGAD, Solanum lycopersicum (BAG16479); HvGAD, Hordeum vulgare (AAV65329); OsGAD, Oryza sativa Japonica Group (BAB32868); NtGAD, Nicotiana tabacum (AAC39483); GmGAD, Glycine max (AAP85548); LjGAD, Lotus japonicus (CAG30580); PtGAD, Populus tremula (ABA18652); AtGAD, Arabidopsis thaliana (NP_197235); BjGAD, Brassica juncea (AAS79672); PpGAD, Pinus pinaster (ABA18653); CsGAD, Citrus sinensis (AAZ05070).

level of ethylene production in naturally ripened bananas. Impressively, a 30-fold sharp increase in endogenous ethylene production (from 0.9 to 29.0) occurred between 0 (FG) and 3 DPH (MY). Moreover, the highest level of endogenous ethylene production in ethylene-treated bananas (29.0) was higher than the highest level in naturally ripened bananas (20.7; Fig. 3). Along the same lines, it took bananas only $6 \mathrm{~d}$ to reach the YB stage, 

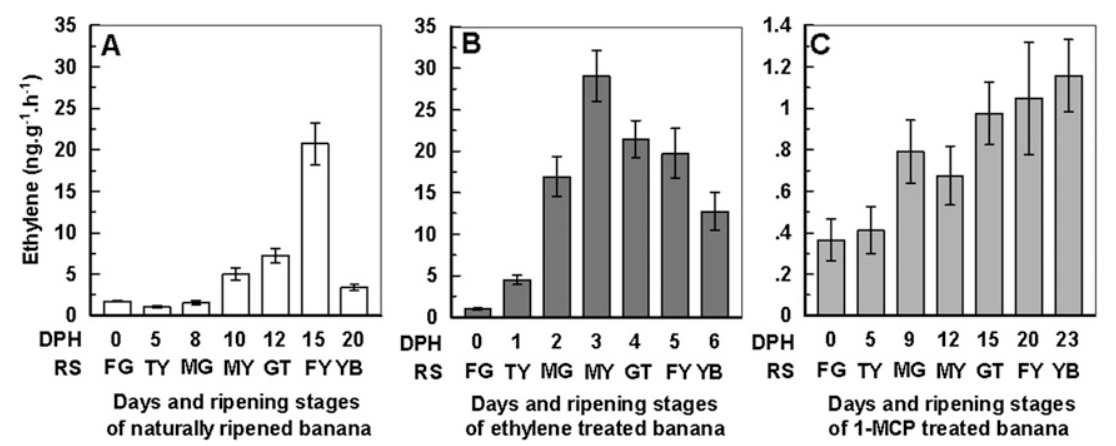

Fig. 3. Changes in ethylene production in naturally ripened (A), ethylene-treated (B) and 1-methylcyclopropene (1-MCP)-treated (C) bananas according to postharvest ripening stages (RS) measured by gas chromatograph. DPH indicates days post-harvest. Vertical bars indicate the SD of three independent experiments.
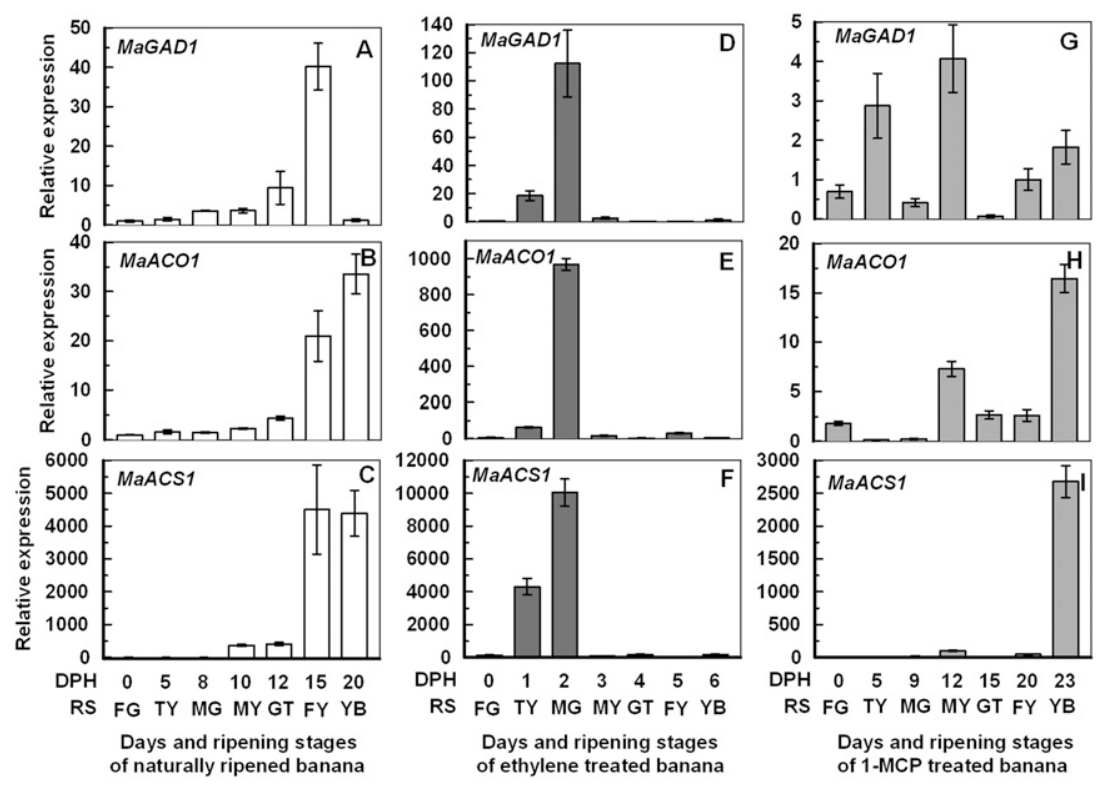

Fig. 4. Expression of $M u G A D, M a A C O 1$, and $M a A C S 1$ in naturally ripened (A-C), ethylene-treated (DF), and 1-methylcyclopropene (1-MCP)-treated (G-I) banana pulp tissue according to the postharvest ripening stages (RS) by real-time reverse transcription-polymerase chain reaction. The y-axis represents the relative fold difference in mRNA level and was calculated with MaActinl as reference. The mRNA fold difference was relative to that of fruit at FG in naturally ripened banana. Vertical bars indicate the SD of three independent experiments.

which normally takes $20 \mathrm{~d}$ with natural ripening (Fig. 3). The expression of $M u G A D$, $M a A C O 1$, and MaACS1 was greatly increased at $1 \mathrm{DPH}(\mathrm{TY})$, and peaked at $2 \mathrm{DPH}(\mathrm{MG})$ with the increase in climacteric ethylene, $\approx 13 \mathrm{~d}$ before the highest levels of expression observed during natural ripening (Fig. 4D-F). Moreover, the actual levels of expression were highly increased by exogenous ethylene treatment. For example, a 293-fold increase in $M u G A D$ expression (from 0.3 to 112.3 ) occurred between 0 (FG) and 2 DPH (MG) (Fig. 4D). Similarly, a 78-fold and 192-fold increase in MaACS1 and MaACO1 expression (128.1 to 10051.3 and 5.0 to 969.8 , respectively) occurred between 0 (FG) and 2 DPH (MG), respectively (Fig. 4E-F).

On the other hand, bananas treated with 1-MCP barely had any detectable ethylene production with a mild increase from 0.3 to 1.1. They also took more time to reach YB stage (23 DPH) and had low levels of $M u G A D$ (from 0.4 to 4.0), MaACO1 (from 0.1 to 16.4), and MaACS1 (form 4.2 to 2678.2) expression compared with naturally ripened bananas during the postharvest ripening progress (Figs. 3 and 4G-I). These results suggested that $M u G A D$ transcript is consistent with endogenous ethylene release and the expression of MaACOI and MaACS1 in naturally ripened, exogenous ethylene-treated and 1-MCP-treated bananas.

\section{Discussion}

Biochemical and physiological changes in banana fruit during postharvest ripening appear to be associated with the activation of a number of genes. Some of these genes such as MaACS1, MaACO1, and malate synthase gene (MaMS1) are related to ethylene biosynthesis, which itself is associated with fruit ripening (Liu et al., 1999; Pua et al., 2003). However, the isolation and characterization of genes encoding banana GAD have not been reported, although GAD plays a role in ethylene biosynthesis (Kathiresan et al., 1997). Moreover, the function of GAD in climacteric ethylene biosynthesis and postharvest fruit ripening is unclear. Here, we report that the isolation and characterization of a gene encoding GAD is involved in climacteric ethylene biosynthesis and fruit ripening in postharvest banana.

Ethylene is an important regulatory factor in fruit ripening, which is a well-documented fact in bananas and other climacteric fruits (Lelièvre et al., 1997; Marriott, 1980). $1-\mathrm{MCP}$ is an antagonist of ethylene that binds to the ethylene receptors (Sisler et al., 1996). The effect of ethylene and 1-MCP on regulating banana fruit ripening has been studied extensively (Golding et al., 1998; Inaba et al., 2007; Mainardi et al., 2006; Pua et al., 2003). Previous studies have shown that MaACS1 and MaACO1 play crucial roles in ethylene biosynthesis during banana postharvest ripening, each having a different role at different ripening stages (Liu et al., 1999). To address the question whether $M u G A D$ expression is involved in ethylene biosynthesis associated with postharvest banana ripening, we used exogenous ethylene and 1-MCP to manipulate this process and measure the rate of endogenous ethylene production, timing of ripening, and $M u G A D, M a A C S 1$, and $M a A C O 1$ expression. Our results showed that coincidently with the ethylene climacteric, the expression level of these genes greatly increased in naturally ripened and ethylenetreated bananas. After ethylene climacteric, $M a A C S 1$ and MaACO1 retained high levels of expression, whereas $M u G A D$ expression decreased sharply in naturally ripened bananas (Figs. 3 and 4). This result implied that coincidently with a long ripening process of naturally ripened bananas, MaACSI and MaACO1 remain playing a role to promote banana fruit ripening after ethylene climacteric, but $M u G A D$ mainly functions in climacteric ethylene biosynthesis. Additionally, $M a A C S 1, M a A C O 1$, and $M u G A D$ expression decreased sharply after ethylene climacteric in bananas treated with exogenous ethylene application (Figs. 3 and 4). This result implied that exogenous ethylene application has a more profound effect on postharvest bananas ripening during the climacteric process, which accelerates the ripening process of postharvest bananas and hence results in low levels of these genes expression after ethylene climacteric.

Moreover, ethylene production, fruit ripening, and gene expression were enhanced by exogenous ethylene application, but inhibited by 1-MCP application (Figs. 3 and 4). Thus, $M u G A D$ expression correlates with ethylene production, fruit ripening, and the expression of MaACS1 and MaACO1, suggesting that $M u G A D$ is involved in climacteric ethylene biosynthesis and fruit ripening in banana fruits after harvest. Although the relationship between GAD activity and ethylene 
production was investigated in excised sunflower tissue (Kathiresan et al., 1997), our study is the first one to explicitly establish a correlation between $M u G A D$ and climacteric ethylene biosynthesis in the postharvest banana ripening process. These findings are supported by our previous cDNA microarray results (Jin et al., 2009; Xu et al., 2007).

In conclusion, ethylene induces the expression of MuGAD, MaACS1, MaACO1, and the postharvest banana ripening process, whereas 1-MCP inhibits these events. MuGAD expression behaves similarly as MaACS1 and MaACO1. Therefore, the findings of this study demonstrated that $M u G A D$ is involved in climacteric ethylene biosynthesis and the fruit ripening process in banana after harvest. GAD is responsible for converting L-glutamic acid to GABA in the presence of the cofactor PLP. GABA was reported to cause an increase in ethylene production rate and up-regulating ACS gene expression (Kathiresan et al., 1997). MuGAD expression correlates with the expression of MaACS1 and MaACO1, implying that GABA produced by $M u G A D$ expression may play a crucial role in ethylene biosynthesis and the fruit ripening process in banana after harvest. In future work, we will put emphasis on characterizing the roles of $M u G A D$ and GABA in ethylene biosynthesis and the fruit ripening process.

\section{Literature Cited}

Adams, D.O. and S.F. Yang. 1979. Ethylene biosynthesis: Identification of 1-aminocyclopropane1-carboxylic acid as an intermediate in the conversion of methionine to ethylene. Proc. Natl. Acad. Sci. USA 76:170-174.

Akama, K., T. Akihiro, M. Kitaqawa, and F. Takaiwa. 2001. Rice (Oryza sativa) contains a novel isoform of glutamate decarboxylase that lacks an authentic almodulin-binding domain at the C-terminus. Biochim. Biophys. Acta 1522:143-150.

Akihiro, T., S. Koike, R. Tani, T. Tominaga, S. Watanabe, Y. Iijima, K. Aoki, D. Shibata, H. Ashihara, C. Matsukura, K. Akama, T. Fujimura, and H. Ezura. 2008. Biochemical mechanism on GABA accumulation during fruit development in tomato. Plant Cell Physiol. 49:1378-1389.

Bolarin, M.C., A. Santa-Cruz, E. Cayuela, and F. PerezAlfocea. 1995. Short-term solute changes in leaves and roots of cultivated and wild tomato seedlings under salinity. J. Plant Physiol. 147:463-468.

Bown, A.W. and B.J. Shelp. 1989. The metabolism and physiological roles of 4-aminobutyric acid. Biochemistry 8:21-25.

Bown, A.W. and B.J. Shelp. 1997. The metabolism and functions of $\gamma$-aminobutyric acid. Plant Physiol. 115:1-5.

Cercós, M., G. Soler, D.J. Iglesias, J. Gadea, J. Forment, and M. Talón. 2006. Global analysis of gene expression during development and ripening of Citrus fruit flesh. A proposed mechanism for citric acid utilization. Plant Mol. Biol. 62:513-527.
Chen, Y., G. Baum, and H. Fromm. 1994. The 58-kilodalton calmodulin-binding glutamate decarboxylase is a ubiquitous protein in petunia organs and its expression is developmentally regulated. Plant Physiol. 106:1381-1387.

Crawford, L.A., A.W. Bown, K.E. Breitkreuz, and F.C. Guinel. 1994. The synthesis of $\gamma$-aminobutyric acid in response to treatments reducing cytosolic pH. Plant Physiol. 104:865-871.

Ford, Y.Y., R.G. Ratcliffe, and R.J. Robins. 1996. Phytohormone-induced GABA production in transformed root cultures of Datura stramonium: An in vivo ${ }^{15} \mathrm{~N}$ NMR study. J. Expt. Bot. 47:811-818.

Gallego, P.P., L. Whotton, S. Picton, D. Grierson, and J.E. Gray. 1995. A role for glutamate decarboxylase during tomato ripening: The characterisation of a cDNA encoding a putative glutamate decarboxylase with a calmodulinbinding site. Plant Mol. Biol. 27:1143-1151.

Golding, J.B., D. Shearer, S.G. Wyllie, and W.B. McGlasson. 1998. Application of 1-MCP and propylene to identify ethylene-dependent ripening processes in mature banana fruit. Postharvest Biol. Technol. 14:87-98.

Hill, S.A. and T. Rees. 1995a. The effect of glucose on the control of carbohydrate metabolism in ripening bananas. Planta 196:335-343.

Hill, S.A. and T. Rees. 1995b. The effect of hypoxia on the control of carbohydrate metabolism in ripening bananas. Planta 197:313-323.

Inaba, A., X. Liu, N. Yokotani, M. Yamane, W.J. Lu, R. Nakano, and Y. Kubo. 2007. Differential feedback regulation of ethylene biosynthesis in pulp and peel tissues of banana fruit. J. Expt. Bot. 58:1047-1057.

Inaba, A. and R. Nakamura. 1986. Effect of exogenous ethylene concentration and fruit temperature on the minimum treatment time necessary to induce ripening in banana fruit. J. Jpn. Soc. Hortic. Sci. 55:348-354.

Jacob-Wilk, D., D. Holland, E.E. Goldschmidt, J. Riov, and Y. Eyal. 1999. Chlorophyll breakdown by chlorophyllase: Isolation and functional expression of the chlase1 gene from ethylene-treated Citrus fruit and its regulation during development. Plant J. 20:653-661.

Jin, Z.Q., B.Y. Xu, J.H. Liu, W. Su, J.B. Zhang, X.L. Yang, C.H. Jia, and M.Y. Li. 2009 Identification of genes differentially expressed at the onset of the ethylene climacteric in banana. Postharvest Biol. Technol. 3:307-309.

Kathiresan, A., P. Tung, C.C. Chinnappa, and D.M. Reid. 1997. Gamma-aminobutyric acid stimulates ethylene biosynthesis in sunflower. Plant Physiol. 115:129-135.

Kulkarni, L. and K. Sohonie. 1956. Glutamic acid decarboxylase in legumes. Nature 178:925.

Lelièvre, J.M., A. Latchè, B. Jones, M. Bouzayen, and J.C. Pech. 1997. Ethylene and fruit ripening. Physiol. Plant. 101:727-739.

Ling, V., W.A. Snedden, B.J. Shelp, and S.M. Assmann. 1994. Analysis of a soluble calmodulin binding protein from fava bean roots: Identification of glutamate decarboxylase as a calmodulin-activated enzyme. Plant Cell 6:1135-1143.

Liu, X., S. Shiomi, A. Nakstsuka, Y. Kubo, R. Nakamura, and A. Inaba. 1999. Characterization of ethylene biosynthesis associated with ripening in banana fruit. Plant Physiol. 121:12571265.

Livak, K.J. and T.D. Schmittgen. 2001. Analysis of relative gene expression data using real-time quantitative PCR and the $2^{-\Delta \Delta \mathrm{Ct}}$ method. Methods 25:402-408.

Lohani, S., P.K. Trivedi, and P. Nath. 2004. Changes in activities of cell wall hydrolases during ethylene-induced ripening in banana: Effect of 1-MCP, ABA and IAA. Postharvest Biol. Technol. 31:119-126.

Mainardi, J.A., E. Purgatto, A. Vieira, W.A. Bastos, B.R. Cordenunsi, and J.R. Oliveira do Nascimento. 2006. Effects of ethylene and 1-methylcyclopropene (1-MCP) on gene expression and activity profile of alpha-1,4glucan-phosphorylase during banana ripening. J. Agr. Food Chem. 54:7294-7299.

Marriott, J. 1980. Bananas-Physiology and biochemistry of storage and ripening for optimum quality. Crit. Rev. Food Sci. Nutr. 13:41-88.

Mayer, R.R., J.H. Cherry, and D. Rhodes. 1990. Effects of heat shock on amino acid metabolism of cowpea cells. Plant Physiol. 94:796-810.

Mazzucotelli, E., A. Tartari, L. Cattivelli, and G. Forlani. 2006. Metabolism of gammaaminobutyric acid during cold acclimation and freezing and its relationship to frost tolerance in barley and wheat. J. Expt. Bot. 57:3755-3766.

Pua, E.C., S. Chandramouli, P. Han, and P. Liu. 2003. Malate synthase gene expression during fruit ripening of Cavendish banana (Musa acuminata cv. Williams). J. Expt. Bot. 54:309-316.

Ramputh, A.L. and A.W. Bown. 1996. Rapid [gamma]-aminobutyric acid synthesis and the inhibition of the growth and development of oblique-banded leaf-roller larvae. Plant Physiol. 4:1349-1352.

Sisler, E.C., M. Serek, and E. Dupille. 1996. Comparison of cyclopropene, 1-methylcyclopropene, and 3,3-dimethylcyclopropene as ethylene antagonists in plants. Plant Growth Regulat. 18:169-174.

Thomas, P. and M.T. Janave. 1992. Effect of temperature on chlorophyllase activity, chlorophyll degradation, and carotenoids of Cavendish bananas during ripening. Intl. J. Food Sci. Technol. 27:57-63.

Tsushida, T. and T. Murai. 1987. Conversion of glutamic acid to $\mathrm{y}$-aminobutyric acid in tea leaves under anaerobic conditions. Agric. Biol. Chem. 51:2865-2871.

Wallace, W., J. Secor, and L.E. Schrader. 1984. Rapid accumulation of $\gamma$-aminobutyric acid and alanine in soybean leaves in response to an abrupt transfer to lower temperature, darkness, or mechanical manipulation. Plant Physiol. 75:170-175.

Wan, C.Y. and T.A. Wilkins. 1994. A modified hot borate method significantly enhance the yield of high-quality RNA from cotton (Gossypium hirsutum L.). Anal. Biochem. 223:7-12.

Xu, B.Y., W. Su, J.H. Liu, J.B. Wang, and Z.Q. Jin. 2007. Differentially expressed cDNAs at the early stage of banana ripening identified by suppression subtractive hybridization and cDNA microarray. Planta 226:529-539.

Yang, S.F. and N.E. Hoffman. 1984. Ethylene biosynthesis and its regulation in higher plants. Annu. Rev. Plant Physiol. 35:155-189. 